\title{
External Application of Herbal Medicines for Acne Vulgaris: A Systematic Review and Meta Analysis
}

\author{
Soo-Hyun Sung ${ }^{1}$, Gwang-Ho Choi², Nam-Woo Lee², Byung-Cheul Shin ${ }^{3 *}$ \\ 1 Department of Policy Development, National Development Institute of Korean Medicine, Seoul, 04554, South Korea \\ ${ }^{2}$ School of Korean Medicine, Pusan National University, Yangsan, 50612, South Korea \\ ${ }^{3}$ Division of Clinical Me dicine, School of Korean Medicine, Pusan National University, Yangsan, 50612, South Korea
}

\section{Key Words}

acne vulgaris, herbal medicine, external application, systematic review, meta-analysis, randomized controlled trial

\begin{abstract}
Aim of the study: The objective of this systematic review is to critically evaluate the evidence of the effectiveness and safety of external application of herbal medicines (EAHM) for acne vurgalis (AV).
\end{abstract}

Methods: English, Chinese and Korean language databases were searched up to May 2018. Randomized clinical trials (RCTs) that reported the effects of EAHM for AV were included and analysed.

Results: A total of 10 randomized trials with $656 \mathrm{AV}$ patients were identified. A meta-analysis of two RCTs indicated that EAHM had a significant effect on improving primary outcome 'global assessment' compared with placebo (mean difference $(\mathrm{MD})=-2.62$, confidence interval $(\mathrm{CI})=-4.84$ to $-0.40, p=0.02$ ). Furthermore, data extracted from two RCTs showed that EAHM significantly reduce primary outcome 'inflammatory lesion count of acne' $(\mathrm{MD}=-1.25, \mathrm{CI}=-1.68$ to $-0.83, \mathrm{p}<0.00001)$ and 'non-inflammatory lesion count of acne' (MD = -1.32, CI $=-1.75$ to $-0.90, \mathrm{p}<0.00001)$. No significant difference was observed between groups in secondary outcome

Received: Oct 01, 2019 Reviewed: Jan 03, 2020 Accepted: Jan 22, 2020

(c) This is an Open-Access article distributed under the terms of the Creative Common Attribution Non-Commercial License (http://creativecommons.org/licenses/by-nc/4.0/) which permits unrestricted noncommercial use, distribution, and reproduction in any medium, provided the original work is properly cited. 'sebum of skin' $(\mathrm{MD}=-0.21, \mathrm{CI}=-0.53$ to $0.11, \mathrm{p}=0.20)$ and 'patient-reported changes in symptom' (relative $\operatorname{risk}(\mathrm{RR})=2.56, \mathrm{CI}=0.43$ to $15.22, \mathrm{p}=0.30$ ). . No severe adverse events (AEs) were found and no treatment was stopped due to AEs of EAHM.

Conclusions: EAHM seems to have affirmative effects, but quality of evidence, and non-standardized use of EAHM make our conclusion weak. Our suggestion is rigorously designed RCTs and standardization of EAHM are required in the future.

\section{Introduction}

Acne vulgaris (AV) is the most common dermatologic disease and affects not only $80 \%$ of adolescents, but also $54 \%$ of adult women and $40 \%$ of adult men [1-3]. The mortality of $\mathrm{AV}$ was not reported, but $\mathrm{AV}$ can cause physical and psychological morbidity such as acne scar, poor self-image, depression and anxiety [4]. In the United States, costs associated with the treatment of AV approximate 3 billion dollars per year [5].

Based on clinical guidelines, the conventional medicines (CM), such as oral medications (e.g., systemic antibiotics, hormonal agents and isotretinoin) and/or topical medications (e.g., benzoyl peroxide, topical antibiotics and topical retinoids), were applied for managing $\mathrm{AV}[4,6,7]$. In a recent published multicenter observational study, topical medications were prescribed for $93 \%$ of $\mathrm{AV}$ patients and oral medications were used for $71 \%$ of cases in Japan [8].

However, these drugs have the potential side effects from local irritation to systemic symptoms including 
liver function abnormalities and teratogenic effects [9]. Traditional herbal medicines have been developed on the basis of unique theories and used to treat various diseases both internally and externally for thousand years [10, 11]. External treatment involves applying drugs on the surface or point of illness [12]. And external application of traditional herbal medicine has been perceived as inexpensive, safer and low cost than CM $[12,13]$.

The recent published systematic review on complementary therapies for AV reported that herbal medicine can be improving symptoms of acne [14]. However, no published systematic reviews have evaluated whether the external application of herbal medicine (EAHM) for AV is safe and effective.

Therefore, we conducted a systematic review following the PRISMA recommendations [15]. The aim of this systematic review is to evaluate the evidence of the effectiveness and safety of the EAHM for AV.

\section{Methods}

\subsection{Protocol and registration}

Our protocol of systematic review was registered in an international prospective register of systematic reviews under the registration number PROSPERO2016:CRD42016050898 (Available from http://www.crd.york.ac.uk/PROSPERO/ display_record.asp?ID=CRD42016050898)

Protocol registration number: CRD42016050898

\subsection{Data Sources and Searches}

We searched the following electronic databases up to May 2018: Pubmed, MEDLINE, EMBASE, the Cochrane Central Register of Controlled Trials (CENTRAL) and CINAHL Plus. We also searched six Korean databases (Korea Institute of Science and Technology Information, Korean traditional knowledge portal, KoreaMed, OASIS, RISS and the National Library of Korea) and two Chinese databases (CNKI and Wanfang). We did not limit publication languages. Furthermore, we also conducted non-electronic searches of conference proceedings, our own files of articles and nine traditional Korean medical journals (Journal of Korean Medicine, the Journal of Korean Acupuncture and Moxibustion Society, Korean Journal of Acupuncture, Journal of Acupuncture and Meridian Studies, Journal of Pharmacopuncture, Journal of Oriental Rehabilitation Medicine, the Journal of Korean Chuna Manual Medicine for Spine and Nerves, Korean Journal of Oriental Physiology and Pathology and the Journal of Korean Oriental Internal Medicine). References of references were reviewed and grey literature was not fully explored.

The search terms were as follows: "acne vulgaris OR acne" AND "external application OR external treatment OR external use OR topical application OR topical use OR topical treatment OR dermal OR skin OR gel OR ointment OR cream OR spray OR oil OR cosmetic product" AND "herb OR herbal medicine OR plant OR plant extract OR ethnobotany OR traditional Chinese medicine OR traditional Korean medicine OR kampo medicine" AND "randomized controlled trial OR randomized clinical trial" in each database language.

\subsection{Study Selection}

\subsubsection{Types of Studies}

We included parallel or cross-over randomized controlled trials (RCTs) to assess the efficacy of EAHM for AV. NonRCTs, animal studies, survey and reviews were excluded.

\subsubsection{Types of Participants}

Patients within any age and gender group diagnosed with AV and participated in the RCTs were included. RCTs applying EAHM in healthy persons were excluded.

\subsubsection{Types of Interventions}

EAHM interventions as any type of intervention in which herbal medicine ingredients were applied to illness area were eligible for inclusion. There is no limitation on the number of herbs, dosage or duration of treatment. We defined herbal medicines as any types of products that originated from botanical sources such as whole plants or their adjuncts [16]. We excluded studies that EAHM interventions were orally administered. We also excluded trials on the combined effects of EAHM and other interventions (e.g., EAHM plus oral administration of herbal medicine, acupuncture or $\mathrm{CM})$.

\subsubsection{Types of Comparisons}

Clinical trials comparing EAHM with placebo or CM were included. CM included zinc sulphate solution, benzoyl peroxide gel. Unqualified control interventions (e.g., herbal medicine) were excluded because their efficacy was not proven.

\subsubsection{Types of Outcome Measures}

The primary outcomes were physician-assessed acne lesion count and physician-assessed global assessment. The secondary outcomes considered in this review were the sebum of skin, moisture of skin, patient-reported changes in symptom, quality of life, anti-bacterial activity test and adverse events (e.g. itching, irritation, erythema and desquamation).

\subsection{Data Extraction}

Three authors (S.H. Sung, G.H. Choi and N.W. Lee) independently screened and selected the included studies and extracted data according to the predefined data extraction form. Data consisted of composition of herbs, used form and amount of EAHM, sample size, EAHM treatment regimens and comparators, outcome measures, reported results and adverse events. Insufficient outcome date were obtained by contacting corresponding authors whenever possible. Disagreements were resolved by discussion among authors (S.H. Sung and B.C. Shin) to reach consensus. 


\subsection{Assessment of Risk of Bias (ROB)}

We used the method for assessment using Cochrane Collaboration's risk of bias tool [17]. This tool includes seven domains, but we assessed the ROB including random sequence generation, allocation concealment, blinding of participants or personnel, blinding of assessors, incomplete outcome data and selective outcome reporting. Two independent authors (S.H. Sung and G.H. Choi) assessed the risk of bias in each study. Any disagreements were resolved by discussion or consultation with a third author (B.C. Shin).

\subsection{Data Analysis}

We used Review Manager (RevMan) software (Version 5.3.5 for windows; the Nordic Cochrane centre, Copenhagen, Denmark) to conduct the meta-analysis. Dichotomous data were presented as risk ratios (RRs) with 95\% confidence intervals (CIs) and continuous outcomes were expressed as mean differences (MDs) with 95\% CIs. Ran- dom-effects model was used in order to combine data into RRs or MDs. I2 tests were used to address the heterogeneity among the included studies. An I2 values $>50 \%$ or $\mathrm{P}$ values $<0.10$ were considerable heterogeneity among studies.[17] A summary of the findings was presented in the results when statistical pooling was not assessed.

\section{Results}

\subsection{Study Selection and Description}

Our search generated a total of 312 potentially relevant studies, finally, 10 RCTs (English databases: $\mathrm{n}=6$; Chinese databases: $\mathrm{n}=1$; Korean databases: $\mathrm{n}=3$ ) [18-27] met our inclusion criteria (Fig. 1). The characteristics of the included RCTs are shown in Table 1. We converted each name of herb into the scientific name.

Three $[18,20,23]$ of the 10 trials were conducted in Korea and published in Korean. For the remaining seven studies, two were conducted in Iraq and published in English [25, 26], and five were conducted in Italy [19], Iran [21], Pakistan [22], India [24] and China [27], respectively.

Table 1 Characteristics of the included RCTs for acne vulgaris

\begin{tabular}{|c|c|c|c|c|c|c|c|}
\hline $\begin{array}{l}\text { First author, } \\
\text { year }\end{array}$ & $\begin{array}{l}\text { Composition } \\
\text { of herb(s), } \\
\text { used form of } \\
\text { EAHM, } \\
\text { amount used }\end{array}$ & $\begin{array}{l}\text { sample size } \\
\text { (randomized } \\
\text { /analysed) }\end{array}$ & $\begin{array}{l}\text { Experimental group } \\
\text { (intervention, regimen) }\end{array}$ & $\begin{array}{l}\text { Control group } \\
\text { (intervention, regimen) }\end{array}$ & Outcome measures & Main results & AEs \\
\hline $\begin{array}{l}\text { Baek, } \\
2011[18]\end{array}$ & $\begin{array}{l}\text { S. flavescens, } \\
\text { Lotion, n.r. }\end{array}$ & $30 / 30$ & $\begin{array}{l}\text { (A) EAHM lotion } \\
\text { (containing } S \text {. flavescens), } \\
\mathrm{n}=15,56 \text { sessions ( } 2 \text { times } \\
\text { per day for } 28 \text { days) }\end{array}$ & $\begin{array}{l}\text { (B) Placebo lotion } \\
\text { (containing } S \text {. flavescens), } \\
\mathrm{n}=15,56 \text { sessions }(2 \text { times } \\
\text { per day for } 28 \text { days) }\end{array}$ & $\begin{array}{l}\text { (1) Sebum of skin } \\
\text { (2) Anti-bacterial } \\
\text { activity test }\end{array}$ & $\begin{array}{l}\text { (1) Significant } \\
\text { difference in }(A)^{a} \\
\text { but not in (B) } \\
\text { (2) NS }\end{array}$ & $\begin{array}{l}\text { Itching (1 in group }(\mathrm{A}) \text { ), } \\
\text { irritation ( } 1 \text { in group } \\
(\mathrm{A}))\end{array}$ \\
\hline $\begin{array}{l}\text { Capitanio, } \\
2012[19]\end{array}$ & $\begin{array}{l}\text { L. digitata, } \\
\text { Cream, n.r. }\end{array}$ & $60 / 60$ & $\begin{array}{l}\text { (A) EAHM cream } \\
\text { (containing } L \text {. digitata), } \\
\mathrm{n}=30,102 \text { sessions }(2 \\
\text { times per day for } 8 \text { weeks) }\end{array}$ & $\begin{array}{l}\text { (A) Placebo cream } \\
\text { (without } L \text {. digitata), } \\
\mathrm{n}=30,102 \text { sessions }(2 \\
\text { times per day for } 8 \text { weeks) }\end{array}$ & $\begin{array}{l}\text { (1) Acne lesion count } \\
\text { (1) Inflammatory lesions } \\
\text { (2) Non- Inflammatory } \\
\text { lesions } \\
\text { (2) Sebum of skin }\end{array}$ & $\begin{array}{l}\text { (1) } \\
\text { (1) (A) better than } \\
\text { (B) but NS } \\
\text { (2) Positive }{ }^{\text {b }} \\
\text { (2) Improved in } \\
\text { (A) and (B) but } \\
\text { NS between } \\
\text { groups }\end{array}$ & None \\
\hline $\begin{array}{l}\mathrm{Du}, \\
2004[20]\end{array}$ & $\begin{array}{l}\text { H. cordata, } \\
\text { Pack, n.r. }\end{array}$ & $65 / 44$ & $\begin{array}{l}\text { (A) EAHM pack } \\
\text { (containing } H \text {. cordata), } \\
\mathrm{n}=24,56 \text { sessions ( } 2 \text { times } \\
\text { per day for } 28 \text { days) }\end{array}$ & $\begin{array}{l}\text { (B) Placebo pack (without } \\
H \text {. cordata), } \mathrm{n}=20,56 \\
\text { sessions ( } 2 \text { times per day } \\
\text { for } 28 \text { days) }\end{array}$ & $\begin{array}{l}\text { (1) Acne lesion count } \\
\square \text { Inflammatory lesions } \\
\square \text { Non- Inflammatory } \\
\text { lesions } \\
\text { (2) patient-reported } \\
\text { changes in symptom }\end{array}$ & $\begin{array}{l}\text { (1) } \\
\text { (1) Significant } \\
\text { difference in }(A)^{\mathrm{c}} \\
\text { but not in }(\mathrm{B}) \\
\text { (2) Significant } \\
\text { difference in }(\mathrm{A})^{\mathrm{c}} \\
\text { but not in (B) } \\
\text { (2) Positive }\end{array}$ & $\begin{array}{l}\text { Itching ( } 2 \text { in group(A)), } \\
\text { irritation ( } 2 \text { in group } \\
\text { (A)), pigmentation ( } 1 \text { in } \\
\text { group (A)) }\end{array}$ \\
\hline $\begin{array}{l}\text { Enshaieh, } \\
2007[21]\end{array}$ & $\begin{array}{l}\text { M. alternifolia, } \\
\text { Gel, n.r. }\end{array}$ & $60 / 60$ & $\begin{array}{l}\text { (A) EAHM gel } \\
\text { (containing } M \text {. alternifolia), } \\
\mathrm{n}=30,90 \text { sessions ( } 2 \text { times } \\
\text { per day for } 45 \text { days) }\end{array}$ & $\begin{array}{l}\text { (B) Placebo gel (without } \\
\text { M. alternifolia), } \mathrm{n}=30,90 \\
\text { sessions ( } 2 \text { times per day } \\
\text { for } 45 \text { days) }\end{array}$ & $\begin{array}{l}\text { (1) Acne lesion count } \\
\text { (1) Inflammatory lesions } \\
\text { (papules) } \\
\text { (2) Inflammatory lesions } \\
\text { (pustules) } \\
\text { (3) Non-inflammatory } \\
\text { lesions (comedones) } \\
\text { (2) Global assessment }\end{array}$ & $\begin{array}{l}\text { (1) } \\
\text { (1) Positive }{ }^{\mathrm{a}} \\
\text { (2) Positive } \mathrm{e}^{\mathrm{b}} \\
\text { (3) Positive }{ }^{\mathrm{c}} \\
\text { (2) Positive }\end{array}$ & $\begin{array}{l}\text { Pruritus ( } 3 \text { in group } \\
\text { (A), } 2 \text { in group (B)), burni } \\
\text { ng sensation (1 in } \\
\text { group (A), } 2 \text { in group } \\
\text { (B)), } \\
\text { scaling ( } 1 \text { in group(A)) }\end{array}$ \\
\hline $\begin{array}{l}\text { Khan, } \\
2014[22]\end{array}$ & $\begin{array}{l}\text { H. rhamnoides, } \\
\text { Emulsion, } \\
\text { total } 500 \mathrm{mg}\end{array}$ & $50 / 50$ & $\begin{array}{l}\text { (A) EAHM emulsion } \\
\text { (containing } H . \text { rhamnoides), } \\
\mathrm{n}=25^{\mathrm{d}}, 112 \text { sessions }(2 \\
\text { times per day for } 8 \text { weeks) }\end{array}$ & $\begin{array}{l}\text { (B) Placebo emulsion } \\
\text { (without plant extract), } \\
\mathrm{n}=25^{\mathrm{d}}, 112 \text { sessions }(2 \\
\text { times per day for } 8 \text { weeks) } \\
\text { (c) EAHM emulsion } \\
\text { (containing C. fistula), } \\
n=25^{d}, 112 \text { sessions }(2 \\
\text { times per day for } 8 \text { weeks) }\end{array}$ & $\begin{array}{l}\text { (1) Global assessment } \\
\text { (2) Sebum of skin } \\
\text { (3) Anti-bacterial } \\
\text { activity test }\end{array}$ & $\begin{array}{l}\text { (1) Unclear } \\
\text { (2) }(A)^{a},(B)^{a} \\
\text { significantly } \\
\text { better than (C) } \\
\text { (3) Anti-bacterial } \\
\text { activity was } \\
\text { observed in (A) } \\
\text { and (B) but not in } \\
\text { (C) }\end{array}$ & $\begin{array}{l}\text { Itching and irritation(4 in } \\
\text { group (A), } 7 \text { in group } \\
\text { (B)) }\end{array}$ \\
\hline $\begin{array}{l}\text { Park, } \\
2013[23]\end{array}$ & $\begin{array}{l}\text { B. platyphyllae, } \\
\text { Foaming cleanser } \\
\text { total } 170 \mathrm{ml} \text {, } \\
\text { toner total } 200 \mathrm{ml}, \\
\text { ampule total } 60 \mathrm{ml}\end{array}$ & $60 / 60$ & $\begin{array}{l}\text { (A) EAHM product } \\
\text { (containing } \quad \text { B. platyphyllae), } \\
\mathrm{n}=30,102 \text { sessions }(2 \\
\text { times per day for } 56 \text { days) }\end{array}$ & $\begin{array}{l}\text { (B) Placebo product } \\
\text { (without } B \text {. platyphyllae), } \\
\mathrm{n}=30,102 \text { sessions ( } 2 \\
\text { times per day for } 56 \text { days) }\end{array}$ & $\begin{array}{l}\text { (1) Global assessment } \\
\text { (2) Sebum of skin } \\
\text { (3) Moisture of skin } \\
\text { (4) Quality of life } \\
\text { (skindex29) }\end{array}$ & $\begin{array}{l}\text { (1) Positive }{ }^{\text {a }} \\
\text { (2) NS } \\
\text { (3) NS } \\
\text { (4) NS }\end{array}$ & None \\
\hline
\end{tabular}




\begin{tabular}{|c|c|c|c|c|c|c|c|}
\hline $\begin{array}{l}\text { Parveen, } \\
2009[24]\end{array}$ & $\begin{array}{l}13 \text { herbs }^{\mathrm{e}} \\
\text { Cream, n.r. }\end{array}$ & $30 / 30$ & $\begin{array}{l}\text { (A) EAHM cream, } n=20 \\
120 \text { sessions ( } 2 \text { times per } \\
\text { day for } 60 \text { days) }\end{array}$ & $\begin{array}{l}\text { (B) Placebo cream, } n=10 \text {, } \\
120 \text { sessions ( } 2 \text { times per } \\
\text { day for } 60 \text { days) }\end{array}$ & (1) Global assessment & $\begin{array}{l}\text { (1) Significant } \\
\text { difference in }(\mathrm{A})^{\mathrm{a}} \\
\text { but not in }(\mathrm{B})\end{array}$ & n.r. \\
\hline $\begin{array}{l}\text { Sharquie, } \\
2006[25]\end{array}$ & $\begin{array}{l}\text { C. sinensis, } \\
\text { Lotion, n.r. }\end{array}$ & $60 / 49$ & $\begin{array}{l}\text { (A) EAHM lotion } \\
\text { (containing C. sinensistea } \\
\text { leaves), } n=25,60 \text { sessions } \\
\text { ( } 2 \text { times per day for } 30 \\
\text { days) }\end{array}$ & $\begin{array}{l}\text { (B) Placebo } \\
\text { solution(without C. } \\
\text { sinensistea leaves), } n=24 \text {, } \\
60 \text { sessions ( } 2 \text { times per } \\
\text { day for } 30 \text { days) }\end{array}$ & $\begin{array}{l}\text { (1) Acne lesion count } \\
\text { (1) Inflammatory lesions } \\
\text { (papules) } \\
\text { (2) Inflammatory lesions } \\
\text { (pustules) } \\
\text { (2) patient-reported } \\
\text { changes in symptom }\end{array}$ & $\begin{array}{l}\text { (1) } \\
\text { (1) Positive } \\
\text { (2) Positive } \\
\text { (2) Positive }\end{array}$ & None \\
\hline $\begin{array}{l}\text { Sharquie, } \\
2008[26]\end{array}$ & $\begin{array}{l}\text { C. sinensis, } \\
\text { Lotion, n.r. }\end{array}$ & $47 / 40$ & $\begin{array}{l}\text { (A) EAHM lotion } \\
\text { (containing C. sinensis), } \\
n=20,120 \text { sessions }(2 \\
\text { times per day for } 60 \text { days) }\end{array}$ & $\begin{array}{l}\text { (B) Zinc sulphate solution, } \\
\mathrm{n}=20,120 \text { sessions ( } 2 \\
\text { times per day for } 60 \text { days) }\end{array}$ & $\begin{array}{l}\text { (1) Global assessment } \\
\text { (2) Acne lesion count } \\
\text { (1) Inflammatory lesions } \\
\text { (papules) } \\
\text { (2) Inflammatory lesions } \\
\text { (pustules) }\end{array}$ & $\begin{array}{l}\text { (1) (A) better than } \\
\text { (B) } \\
\text { (2) } \\
\text { (1) Significant } \\
\text { difference in }(A)^{c} \\
\text { but not in (B) } \\
\text { (2) Significant } \\
\text { difference in }(A)^{c} \\
\text { but not in (B) }\end{array}$ & $\begin{array}{l}\text { Itching ( } 5 \text { in group } \\
\text { (A), } 2 \text { in group (B)), burni } \\
\text { ng sensation ( } 5 \text { in } \\
\text { group (B)) }\end{array}$ \\
\hline $\begin{array}{l}\text { Zeng, } \\
2012[27]\end{array}$ & $\begin{array}{l}\text { S. baicalensis } \\
+ \text { P. amurense } \\
+ \text { I. tincoria, } \\
\text { Pack, n.r. }\end{array}$ & $233 / 233$ & $\begin{array}{l}\text { (A) EAHM pack, } n=113,8 \\
\text { sessions ( } 2 \text { times per week } \\
\text { for } 4 \text { weeks)bs }\end{array}$ & $\begin{array}{l}\text { (B) Benzoyl peroxide gel, } \\
n=120,28 \text { sessions ( } 1 \text { time } \\
\text { per day for } 28 \text { days) }\end{array}$ & $\begin{array}{l}\text { (1) Acne lesion count } \\
\text { (2) patient-reported } \\
\text { changes in symptom }\end{array}$ & $\begin{array}{l}\text { (1) Positive } \text { Pa }^{a} \\
\text { (2) Positive }\end{array}$ & $\begin{array}{l}\text { AEs ( } 2 \text { in group }(\mathrm{A}), 3 \\
\text { in group }(\mathrm{B}))\end{array}$ \\
\hline
\end{tabular}

${ }^{\mathrm{a}} \mathrm{p}<0.05 ;{ }^{\mathrm{b}} \mathrm{p}<0.01 ;{ }^{\mathrm{c}} \mathrm{p}<0.001 ;{ }^{\mathrm{d}}$ cheeks of same patients with acne received (A) versus (C), and (B) versus (C) respectively; ${ }^{\mathrm{e}} 13$ herbs: $A$. barbadensis $+A$. indica $+A$. sarcocolla + B. serrata + C. myrrha + C. rotundus $+C$. zizanoides $+J$. officinale + L. ensata + M. chamomilla $+N$. arbor-tristis $+O$. europaea $+P$. dulcis

A. barbadensis, Aloe barbadensis; A. indica, Azadirachta indica; A. sarcocolla, Astragalus sarcocolla; AEs, Adverse events; B. Platyphyllae, Betulae platyphyllae; $B$. serrata, Boswellia serrata; C. fistula, Cassia fistula; C. myrrha, Commiphora myrrha; C. rotundus, Cyperus rotundus; C. sinensis, Camellia sinensis; C. zizanoides, Chrysopogon zizanoides; EAHM, External application of herbal medicine; H. cordata, Houttuynia cordata; H. rhamnoides, Hippophae rhamnoides; I. ensata, Iris ensata; I. tinctoria, Isatis tinctoria; J. officinale, Jasminum officinale; L. digitate, Laminaria digitata; M. alternifolia, Melaleuca alternifolia; M. chamomilla, Matricaria chamomilla; N. arbor-tristis, Nyctanthes arbor-tristis; NS, No significant difference; O. europaea, Olea europaea; P. amurense, Phellodendron amurense; P. dulcis, Prumus dulcis; S. baicalensis, Scutellaria baicalensis; S. flavescens, Sophora flavescens.



Figure 1 Flowchart of the RCT selection process. CCTs: Controlled Clinical Trial s ; RCTs: Randomized Controlled Trial s ; E AHM: External Application of Herbal Medicine 


\subsection{Participants}

A total of 656 participants were included in 10 trials. The sample sizes in each group ranged from 15 to 113 in the EAHM group and from 10 to 120 in control group. The median sample sizes per arm were 33 in the EAHM group and 32 in the control group. One study [22] reported cheeks of same patients with AV received EAHM (containing Hippophae rhamnoides) versus placebo, and EAHM (containing Cassia fistula) versus placebo respectively.

\subsection{Interventions}

The types of EAHM were very diverse . Eight [18-23, 25, $26]$ reported single herbal medicine products : two studies [25, used Camellia sinensis ; Sophora flavescens [18], Laminaria digitata [19], Houttuynia cordata [20], Melaleuca alternifolia [21], Hippophae rhamnoides [22] and Betulae platyphyllae [23] were used in one study respectively . The other two studies [24, externally applied mixed herbal medicine products on $\mathrm{AV}$ patients : one study [ used unani herbomineral formulation (Aloe barbadensis Azadirachta indica, Astragalus sarcocolla Boswellia serrata Commiphora myrrha Cyperus rotundus Chrysopogon zizanoides Jasminum officinale Iris ensata Matricaria chamomilla Nyctanthes arbor tristis Olea europaea Prunus dulcis); another study [ used Chinese medical formulation Scutellaria baicalensis , Phellodendron amurense , Isatis tinctoria)
Eight trials [18-25] compared EAHM interventions with placebo interventions. The remaining trials, one study [26] compared effects of EAHM to zinc sulphate, and another trial [27] contrasted EAHM treatment to benzoyl peroxide intervention.

\subsubsection{Types of EAHM forms}

Various types of EAHM form were used in 10 included studies. Of these, three studies $[18,25,26]$ applied EAHM lotion to AV patients. Cream $[19,24]$ and pack $[20,27]$ types were utilized in two studies. One study was for gel [21] and emulsion [22] respectively. One study [23] used three types of forms including foaming cleanser, toner and ample, with same ingredients on AV patients.

\subsubsection{Amount of EAHM Used}

The amount of EAHM used was reported in only two studies: one study [22] utilized total $500 \mathrm{mg}$ of EAHM. A total $170 \mathrm{ml}$ of EAHM foaming cleanser, $200 \mathrm{ml}$ of EAHM toner and $60 \mathrm{ml}$ of EAHM ampule were used in the other study [23].

\subsection{Outcome Measures}

Six studies [18-20, 23-25] that placebo was used as a comparison were available for meta-analysis (Fig. 2). We summarized the results of the studies that statistical pooling was not assessable.

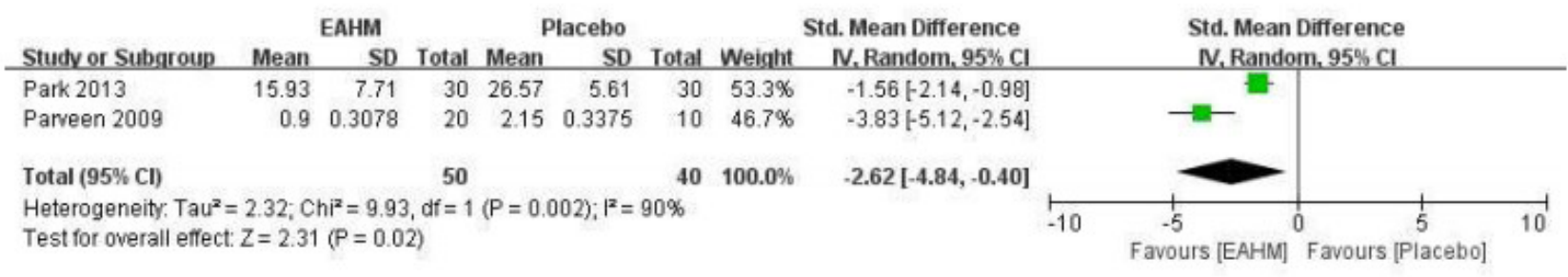

(a) Global assessment



(b) Inflammatory lesion count of acne

\begin{tabular}{|c|c|c|c|c|c|c|c|c|c|c|c|}
\hline \multirow[b]{2}{*}{ Study or Subgroup } & \multicolumn{3}{|c|}{ ЕАНМ } & \multicolumn{3}{|c|}{ Placebo } & \multicolumn{2}{|r|}{ Std. Mean Difference } & \multirow{2}{*}{\multicolumn{3}{|c|}{$\begin{array}{l}\text { Std. Mean Difference } \\
\text { IV. Random. } 95 \% \mathrm{Cl}\end{array}$}} \\
\hline & Mean & SD & Total & Mean & SD & Total & Weight & N. Random, $95 \% \mathrm{Cl}$ & & & \\
\hline Capitanio 2012 & 19.7 & 13 & 30 & 38.4 & 18 & 30 & $60.7 \%$ & $-1.18[-1.73,-0.62]$ & 듬- & & \\
\hline Du 2004 & 3.29 & 2.35 & 24 & 8.5 & 4.16 & 20 & $39.3 \%$ & $-1.55[-2.24,-0.87]$ & & & \\
\hline Total $(95 \% \mathrm{Cl})$ & & & 54 & & & 50 & $100.0 \%$ & $-1.32[-1.75,-0.90]$ & & & \\
\hline $\begin{array}{l}\text { Heterogeneity: Tauª } \\
\text { Test for overall effect }\end{array}$ & $\begin{array}{l}0.00 ; \mathrm{Ch} \\
\mathrm{Z}=6.05\end{array}$ & $\begin{array}{l}\mathrm{n}^{2}=0 . \\
(\mathrm{P}<0\end{array}$ & $\begin{array}{l}71, \mathrm{df}= \\
.00001)\end{array}$ & $=1(P=$ & $0.40) ; 1$ & $F^{2}=0 \%$ & & & $\begin{array}{c}-2 \\
\text { Favours [EAHI }\end{array}$ & ]$^{0}$ Favours IP & 2 \\
\hline
\end{tabular}




\section{(c) Non-inflammatory lesion count of acne}

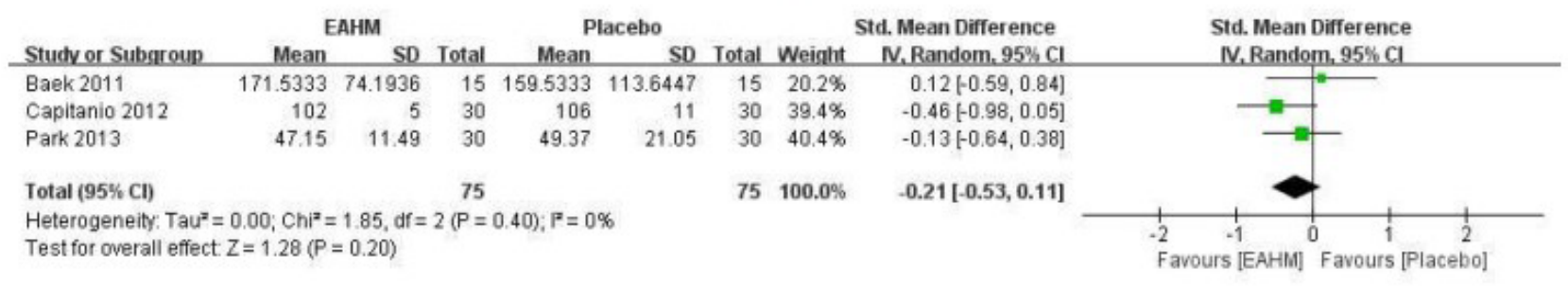

(d) Sebum of skin

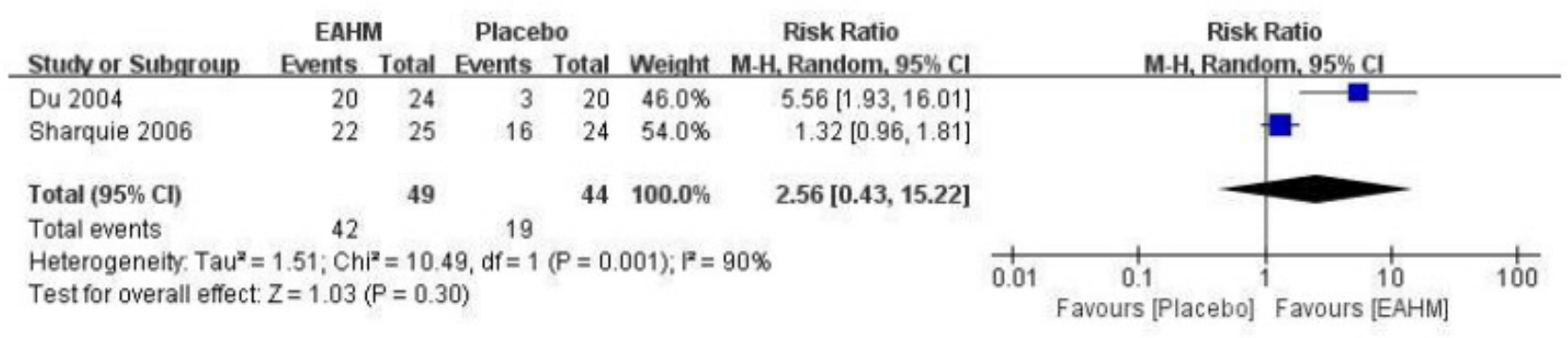

\section{(e) Patient-reported changes in symptom}

Figure 2 Meta analysis of EAHM versus placebo. EAHM; External Application of Herbal

Medicine; CI: Confidence Intervals; SD: Standard Deviation.

\subsubsection{EAHM versus Placebo}

Of the eight studies [18-25] that compared EAHM with placebo interventions, six studies [18-20, 23-25] provided data for statistical pooling.

Four studies [21-24] compared primary outcome 'global assessment' between EAHM and placebo; two studies [23, $24]$ of meta-analysis showed significant effect on improving the global assessment [Fig. 2(a), $\mathrm{MD}=-2.62, \mathrm{CI}=-4.84$ to $-0.40, \mathrm{P}=0.02]$ with high heterogeneity $(\mathrm{P}=0.002, \mathrm{I} 2=$ $90 \%)$; in the study by Enshaieh et al. [21] reported significant difference in two groups $(\mathrm{P}<0.001)$; we did not assess result of one trial due to insufficient data. [22] Park et al. [23] divided face into six parts, and assigned scores for each part.

For primary outcome 'lesion count of acne', data extracted from two studies $[19,20]$ showed a significant superior improvement in EAHM compared to placebo in inflammatory lesion count of acne [Fig. 2(b), MD =-1.25, CI = -1.68 to $-0.83, \mathrm{P}<0.00001$, I2 $=0 \%$ ] and non- inflammatory lesion count of acne [Fig. 2(c), $\mathrm{MD}=-1.32, \mathrm{CI}=-1.75$ to $-0.90, \mathrm{P}<0.00001$, I $2=0 \%]$. As meta-analysis was impossible in the other two trials, we described the result of the studies as follows; one trial [21] showed significant improvement in inflammatory lesion count of papules $(\mathrm{P}$ $<0.05)$, inflammatory lesion count of pustules $(\mathrm{P}<0.01)$ and non-inflammatory lesion count of comedones $(\mathrm{P}<$ 0.001); in another trial [25], EAHM treatment significantly reduced inflammatory lesion count of papules $(\mathrm{P}<0.05)$ and pustules $(\mathrm{P}<0.05)$ compared with placebo.
Among eight studies that contrasted EAHM with placebo, four trials assessed secondary outcome 'sebum of skin'; meta-analysis on three studies $[18,19,23]$ showed no significant difference between the groups [Fig. 2(d), MD = $-0.21, \mathrm{CI}=-0.53$ to $0.11, \mathrm{P}=0.20, \mathrm{I} 2=0 \%]$; in the study by Khan et al. [22] reported EAHM group was more effective than placebo group in sebum of skin $(\mathrm{P}<0.05)$.

The pooled data from two trials $[20,25]$ indicated no significant effect in secondary outcome 'patient-reported changes in symptom' [Fig. $2(\mathrm{e}), \mathrm{RR}=2.56, \mathrm{CI}=0.43$ to $15.22, \mathrm{P}=0.30, \mathrm{I} 2=90 \%]$.

In the trial of Baek et al. [18], the effect was not significant in secondary outcome 'anti-bacterial activity test'. Anti-bacterial activity was observed in EAHM group but not in placebo group.[22] There were no significant differences between groups in secondary outcome 'quality of life'. [23]

\subsubsection{Adverse Events}

Seven studies [18-23, 25] reported AEs; Baek et al. [18] reported minor AEs, such as itching and irritation in EAHM group; we found itching, irritation and pigmentation as minor AEs of EAHM treatment [20]; one trial [21] reported pruritus, burning sensation and scaling occurred in the EAHM group, pruritus and burning sensation presented in the control group; Khan et al. [22] reported four cases of itching and irritation occurred in the EAHM group, and seven cases of itching and irritation presented in the placebo group; AEs did not occur in another three studies [19, $23,25]$. One study [24] did not mention AEs. We pooled 
data from seven trials [18-23, 25], EAHM group reported more AEs than placebo group but no statistically significant differences were observed between groups [Fig. 3(a), $\mathrm{RR}=1.37, \mathrm{CI}=0.46$ to $4.09, \mathrm{P}=0.58, \mathrm{I} 2=39 \%]$.

\begin{tabular}{|c|c|c|c|c|c|c|c|c|c|c|c|}
\hline Study or Subqroup & $\begin{array}{l}\text { EAHM } \\
\text { Events }\end{array}$ & Total & $\begin{array}{l}\text { Placel } \\
\text { Events }\end{array}$ & Total & Weight & $\begin{array}{c}\quad \text { Risk Ratio } \\
\text { M-H. Random. } 95 \% \mathrm{Cl}\end{array}$ & & $\begin{array}{r}\text { Risk I } \\
\text { M-H. Rando }\end{array}$ & $\begin{array}{l}\text { Ratio } \\
\text { om, } 95 \% \mathrm{Cl}\end{array}$ & & \\
\hline Beak 2011 & 2 & 15 & 0 & 15 & $11.4 \%$ & $5.00[0.26,96.13]$ & & & & & \\
\hline Capitanio 2012 & 0 & 30 & 0 & 30 & & Not estimable & & & & & \\
\hline Du 2004 & 5 & 24 & 0 & 20 & $12.2 \%$ & $9.24[0.54,157.57]$ & & & & & \\
\hline Enshaieh 2007 & 5 & 30 & 4 & 30 & $36.6 \%$ & $1.25[0.37,4.21]$ & & & & & \\
\hline Khan 2014 & 4 & 25 & 7 & 25 & $39.9 \%$ & $0.57[0.19,1.71]$ & & & & & \\
\hline Park 2013 & 0 & 30 & 0 & 30 & & Not estimable & & & & & \\
\hline Sharquie 2006 & 0 & 25 & 0 & 24 & & Not estimable & & & & & \\
\hline Total $(95 \% \mathrm{Cl})$ & & 179 & & 174 & $100.0 \%$ & $1.37[0.46,4.09]$ & & & & & \\
\hline Total events & 16 & & 11 & & & & & & & & \\
\hline \multicolumn{7}{|c|}{$\begin{array}{l}\text { Heterogeneity: } \text { Tau }^{2}=0.47 ; \mathrm{Ch}^{2}=4.95, \mathrm{df}=3(P=0.18) ; \mathrm{I}^{2}=39 \% \\
\text { Test for overall effect: } Z=0.56(P=0.58)\end{array}$} & 0.01 & $\begin{array}{c}0.1 \\
\text { Favours [EAHM] }\end{array}$ & Favours [p & $\begin{array}{l}10 \\
\text { placebo] }\end{array}$ & 100 \\
\hline
\end{tabular}

(a) EAHM versus placebo

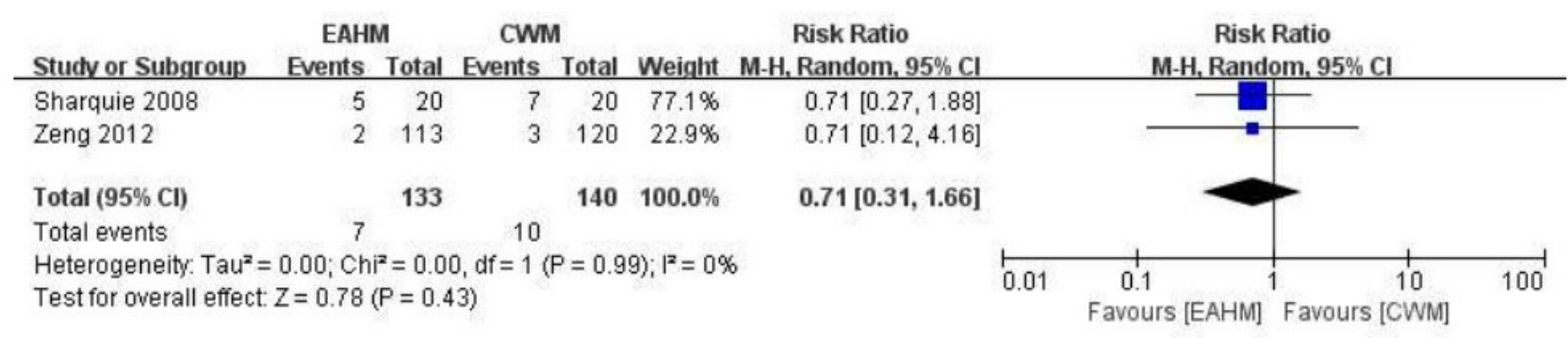

\section{(b) EAHM versus CM}

Figure 3 Meta-analysis of adverse events; EAHM: External Application of Herbal Medicine; CI: Conficence Intervals; CM: Conventional Medication.

\subsubsection{EAHM versus CM}

We summarised the results of two studies [26, 27] compared EAHM with CM intervention because data pooling was not assessable.

Two trials $[26,27]$ evaluated the effectiveness and safety of EAHM compared with CM; in the study by sharquie [26] that compared EAHM lotion (containing Camellia sinensis) with zinc sulphate reported an improvement in global assessment. A significant improvement at acne count of inflammatory lesion $(\mathrm{P}<0.001)$ and non-inflammatory lesion $(\mathrm{P}<0.001)$ were observed in EAHM group but not in control group; the results of study [27] that contrasted EAHM pack with benzoyl peroxide indicated significant difference in acne lesion count and patient-reported changes in symptom $(\mathrm{P}<0.05)$.

\subsubsection{Adverse Events}

There were no severe AEs in all participants; one study [26] reported five cases of itching occurred in the EAHM group, and two cases of itching and five cases of burning sensation represented in the control group; another study [27] mentioned two cases of AEs occurred in the treatment group, and three AEs presented in the control group. The results of AEs did not show a significant difference between the groups (Fig. 3(b), RR $=0.71, \mathrm{CI}=0.31$ to $1.66, \mathrm{P}$ $=0.43, \mathrm{I} 2=0 \%)$.

\subsection{Cochrane risk of bias assessment}

Overall, the included RCTs had a low methodological quality (Table 2). Only one trial [21] reported adequate random sequence generation using computer number generator, whereas two trials $[19,23]$ reported inappropriate method (sequence generated by patients entered the study). 
Allocation was properly concealed in two trials. One study $[19,21]$ reported that allocation was properly conducted using identical appearance that is sealed randomization code. Another study [21] reported that allocation was performed by and independent person.

The participant and practitioner were blinded in five trials [18-21, 23]; Double-blinded RCTs were conducted in two trials $[19,21]$ and same form of intervention was used in the EAHM and control groups. [18, 20, 23] Single-blinded studies were employed in four studies [22, 24-26] and one study [27] used different types of intervention form in both groups. Only one trial [20] reported the details of the blinding of outcome assessment.

Most of the included studies [18, 19, 21, 23, 24-27] had low risk of bias addressing incomplete outcome data (e.g., no missing outcome data or missing outcome data, but the drop-out rate did not exceed $20 \%$ for short-term follow-up). Two trials $[20,22]$ had high ROB; one [20] had missing data and the drop-out rate exceeded $20 \%$ for short-term follow-up, and another [22] reported that the number of patients was different when we compared pre-treatment with post-treatment in outcome measure of global assessment.

Regarding selective outcome reporting, only one [22] studies reported their protocol before conducting the RCTs.

\section{Discussion}

This systematic review examined the effectiveness and safety of EAHM for treating AV. To the best of our knowledge, this is the first systematic review to provide current available evidence about EAHM for managing AV. From the eight studies [18-25] that compared EAHM with placebo, the positive outcomes and meta-analysis for primary outcomes were found. In the results of the studies that contrasted EAHM with CM [26, 27], the treatment group showed more effective than the control group in primary outcomes. The median sample sizes per arm were 33 in the EAHM group and 32 in the control group. All of the included trials involved small sample sizes; there were only one study [27] with $\geq 40$ participants in each group. Based on the results, especially one high quality RCT [21], EAHM appear to improve primary outcome 'acne lesion count' and 'global assessment'. However, it is not conclusive due to the low methodological quality, and small number of included studies.

In terms of safety, more AEs were observed in EAHM groups compare to placebo groups [18-25], the difference between groups was not statistically significant. Fewer AEs reported among patients treated with EAHM relative to CM [26, 27]; however, no significant differences were observed between groups. The goals of acne treatment are to lessen the acne lesions, improve appearance and minimise potential AEs.[28] Finally, EAHM does not appear to be related to severe AEs, suggesting that EAHM might be safe for AV patients. However, the number of RCTs was too small, so this finding should be interpreted with caution.

Although all the included studies stated that the patients were randomly assigned, only one trial [21] used computer randomization and allocation concealment was stated in two trials [19, 21]. Only one study [21] had a low risk of bias for blinding of participants, personnel and outcome assessors. Bias resulting from inadequate random sequence generation, allocation concealment, or lack of blinding was related to over-positive estimates of intervention effects for subjectively assessed outcomes [29, 30]. For this reason, the potential risk of bias should be minimized to accurate evaluation of EAHM interventions. Among 10 included trials, three studies [18, 20, were conducted in Korea. Although various types of complementary and alternative medicine (CAM) RCTs (e.g. herbal medicine, acupuncture, tuina) were published in the Korea, Korean CAM RCTs were generally excluded from systematic review because Korean trials were not indexed in English data bases such as MEDLINE, EMBASE or Cochrane library [ The unbiased search of various databases without a language restriction is important to avoid language bias. The difference that compared active treatment group with placebo group is called the specific effect of that treatment [32]. To measure specific effects, it is necessary to conduct placebo-controlled RCTs [32]. Even though eight studies [18-25] compared EAHM with placebo, the consideration of placebo that had the same level of flavours as the EAHM treatment was not mentioned. The unique flavours

Table 2 Risk of bias assessment.

\begin{tabular}{lcccccc}
\hline \multicolumn{1}{c}{ First author, year } & \multicolumn{2}{c}{ Selection bias } & $\begin{array}{c}\text { Performan } \\
\text { ce bias }\end{array}$ & $\begin{array}{c}\text { Detection } \\
\text { bias }\end{array}$ & $\begin{array}{c}\text { Attrition } \\
\text { bias }\end{array}$ & $\begin{array}{c}\text { Reporting } \\
\text { bias }\end{array}$ \\
\cline { 2 - 7 } & $\begin{array}{c}\text { Random } \\
\text { sequence } \\
\text { generation }\end{array}$ & $\begin{array}{c}\text { Allocation } \\
\text { concealment }\end{array}$ & $\begin{array}{c}\text { Blinding of } \\
\text { participants } \\
\text { and personnel }\end{array}$ & $\begin{array}{c}\text { Blinding of } \\
\text { outcome } \\
\text { assessment }\end{array}$ & $\begin{array}{c}\text { Incomplete } \\
\text { outcome data }\end{array}$ & $\begin{array}{c}\text { Selective } \\
\text { reporting }\end{array}$ \\
\hline Baek, 2011 [18] & $\mathrm{U}$ & $\mathrm{U}$ & $\mathrm{L}$ & $\mathrm{U}$ & $\mathrm{L}$ & $\mathrm{U}$ \\
Capitanio, 2012 [19] & $\mathrm{H}$ & $\mathrm{L}$ & $\mathrm{L}$ & $\mathrm{U}$ & $\mathrm{L}$ & $\mathrm{U}$ \\
Du, 2004 [20] & $\mathrm{U}$ & $\mathrm{U}$ & $\mathrm{L}$ & $\mathrm{U}$ & $\mathrm{H}$ & $\mathrm{U}$ \\
Enshaieh, 2007 [21] & $\mathrm{L}$ & $\mathrm{L}$ & $\mathrm{L}$ & $\mathrm{L}$ & $\mathrm{L}$ & $\mathrm{U}$ \\
Khan, 2014 [22] & $\mathrm{U}$ & $\mathrm{U}$ & $\mathrm{H}$ & $\mathrm{U}$ & $\mathrm{H}$ & $\mathrm{L}$ \\
Park, 2013 [23] & $\mathrm{H}$ & $\mathrm{H}$ & $\mathrm{L}$ & $\mathrm{U}$ & $\mathrm{L}$ & $\mathrm{U}$ \\
Parveen, 2007 [24] & $\mathrm{U}$ & $\mathrm{U}$ & $\mathrm{H}$ & $\mathrm{U}$ & $\mathrm{L}$ & $\mathrm{U}$
\end{tabular}


of herbal medicine are potentially leading to the failure of participant and practitioner blinding.[33] Therefore, future studies should investigate appropriate placebo intervention that considered the identical shape and scent as experimental EAHM.

We reviewed the 10 included studies that used in the diverse types of EAHM form, ingredient and dosage. Although our review presented positive results, the standardisation of EAHM intervention was not performed. Therefore, Standardization of EAHM that considered following factors is necessary to apply EAHM to AV patients; (1) types of EAHM form and ingredient; (2) duration of treatment and number of treatment sessions based on the each EAHM intervention; (3) EAHM dosage for total treatment and one session; (4) Appropriate placebo model. Future trials need to adhere to 'consolidated standards of reporting trials (CONSORT) for traditional Chinese medicine' [34].

Only two studies $[22,23]$ reported total EAHM dosage during treatment. The same EAHM formulations might have different effects according the EAHM dosage. This is due to the lack of dosage guideline of EAHM on AV. For establishing EAHM dosage guideline for AV, further research should be conducted based on these results.

The strength of our review is that we searched various databases without language restriction; thus, researchers could assess papers published in East-Asian (Chinese and Korean). Furthermore, the screening and data extraction of East-Asian studies were conducted by Traditional Korean Medicine (TKM) doctors (S. H. S. and G. H. C.) and TCM doctor (N. W. L.). However, this systematic review has some limitations. From the 10 included RCTs, we conducted meta-analysis of six studies; however, due to clinical heterogeneity of control intervention and outcome measure, we performed statistical pooling of two to three trials for each outcome measures. Also, we analyzed the collected RCTs without setting the definition and scope of herbal medicines. The interpretation of the effectiveness of herbal medicines for AV may vary depending on the definition and scope of herbal medicines. In addition, it is difficult to recommend EAHM as a clinical practice guideline for the treatment of AV because of low methodological quality, small sample size and insufficient information of EAHM dosage.

In Future, lager, more rigorous and adequately powered multi-centre RCTs considering these limitations should be investigated.

\section{Conclusion}

The EAHM seems to have beneficial effectiveness and safe treatment in patients with AV. However, the evidence is insufficient to conclude the effectiveness and safety of EAHM treatment because the analyses were based on a small number of included studies and sample size. Therefore, well-designed, high quality and multi-centre RCTs are needed to provide evidence-based treatment in AV.

\section{Conflicts of interest}

The authors declare that there are no conflicts of interest regarding the publication of this paper.

\section{Authors' Contribution}

S.H. Sung, B.C. Shin, N.W. Lee and G.H. Choi designed the review. S.H. Sung, G.H. Choi and N.W. Lee completed the literature search, study selection and data extraction. S.H. Sung and G.H. Choi evaluated the methodological quality. They were checked by B.C. Shin, G.H. Choi and B.C. Shin performed the meta-analysis. S.H. Sung and G.H. Choi wrote the article. B.C. Shin revised the manuscript. All authors have read and approved the final manuscript.

\section{Financial support}

This work was supported by a grant to Korean Medical Science Research Center for Healthy Aging from the National Research Foundation of Korean government (2014R1A5A2009936).

\section{ORCID}

Soo-Hyun Sung. https://orcid.org/0000-0001-7606-0147 Gwang-Ho Choi. https://orcid.org/0000-0001-6443-5941 Nam-Woo Lee. https://orcid.org/0000-0002-2662-5944 Byung-Cheul Shin. https://orcid.org/0000-0002-00592689

\section{References}

1. Knutsen-Larson S, Dawson AL, Dunnick CA, et al. Acne vulgaris: pathogenesis, treatment, and needs assessment. Dermatol Clin. 2012;30:99-106.

2. Lynn DD, Umari T, Dunnick CA, et al. The epidemiology of acne vulgaris in late adolescence. Adolesc Health Med Ther. 2016;19:13-25.

3. Ramos-e-Silva M, Carneiro SC. Acne vulgaris: review and guidelines. Dermatol Nurs. 2009;21:63-8.

4. Zaenglein AL, Pathy AL, Schlosser BJ, et al. Guidelines of care for the management of acne vulgaris. J Am Acad Dermatol. 2016;74:945-73.

5. Bhate K, Williams HC. Epidemiology of acne vulgaris. Br J Dermatol. 2013;168:474-85.

6. Gollnick HP, Krautheim A. Topical treatment in acne: current status and future aspects. Dermatology. 2003;206:29-36.

7. Arshdeep De D. What's new in the management of acne? Indian J Dermatol Venereol Leprol. 2013;79:279-87.

8. Miyachi Y, Hayashi N, Furukawa F, et al. Acne management in Japan: study of patient adherence. Dermatology. 2011;223:174-81.

9. Tripathi SV, Gustafson CJ, Huang KE, et al. Side effects of common acne treatments. Expert Opin Drug Saf. 2013;12:39-51.

10. Park BG, Jun JH, Jung JY, et al. Herbal medicines for cancer cachexia: protocol for a systematic review. BMJ Open. 2014;4:e005016.

11. Xu Y. Dermatology in traditional Chinese medicine, ed 1. Donica Publishing Ltd(St. Albans). 2004. 
12. Li N, Li YQ, Li HY, et al. Efficacy of externally applied Chinese herbal drugs in treating psoriasis: a systematic review. Chin J Intergr Med. 2012;18:222-9.

13. Li X, Xiao QQ, Ze K, et al. External application of traditional Chinese medicine for venous ulcers: a systematic review and meta-analysis. Evid Based Complement Alternat Med. 2015;2015:831474.

14. Cao H, Yang G, Wang Y, et al. Complementary therapies for acne vulgaris. Cochrane Database Syst Rev. 2015;1:CD009436.

15. Moher D, Liberati A, Tetzlaff J, Altman DG. Preferred reporting items for systematic reviews and meta-analyses: the PRISMA statement. Annals of Internal Medicine. 2009;151:264-9.

16. Ernst E, Pittler MH, Wider B, Boddym K. Oxford handbook of complementary medicine. Oxford University Press(Oxford) 2008.

17. Higgins JPT, Altman DG, Gøtzsche PC, et al. The cochrane collaboration's tool for assessing risk of bias in randomised trials. BMJ. 2011;343:1-9.

18. Baek SC, Jo EH, Mendgerel, Park MC. Effect of Sophorae radix-skin lotion on acne. J Korean Med Ophthalmol Otolaryngol Dermatol. 2011;24:111-20.

19. Capitanio B, Sinagra JL, Weller RB, et al. Randomized controlled study of a cosmetic treatment for mild acne. Clin Exp Dermatol. 2012;37:346-9.

20. Du IS, Seo YJ, Woo WH, et al. Clinical study on the effect of external treatment of 5\% Herba Houttuyniae extract solute on on the acne. Korean J Oriental Physiology \& Pathology. 2004;18:612-20.

21. Enshaieh S, Jooya A, Siadat AH, Iraji F. The efficacy of $5 \%$ topical tea tree oil gel in mild to moderate acne vulgaris: a randomized, double-blind placebo-controlled study. Indian J Dermatol Venereol Leprol. 2007;73:22-5.

22. Khan BA, Akhtar N. Clinical and sebumetric evaluation of topical emulsions in the treatment of acne vulgaris. Postepy Dermatol Alergol. 2014;31:229-34.

23. Park SY, Na CS, Jeong WC, Lee JC. A clinical study for effect of herbal cosmetics containing Cortex betulae platyphyllae extract complex on acne. J Korean Med Ophthalmol Otolaryngol Dermatol. 2013;26:1-19.

24. Parveen S, Zafar S, Qureshi MA, Bano H. Clinical trial of unani herbomineral cream to evaluate its topical effects on acne vulgaris. Indian J Tradit Knowl. 2009;8:431-6.

25. Sharquie KE, Al-Turfi A, Al-Shimary WM, Treatment of acne vulgaris with $2 \%$ topical tea lotion. Saudi Med J 2006;27:83-5.

26. Sharquie KE, Noaimi AA, Al-salih MM. Topical therapy of acne vulgaris using $2 \%$ tea lotion in comparison with 5\% zinc sulphate solution. Saudi Med J. 2008;29:1757-61.

27. Zeng X, Liu WL, Zhao T. Effects of Chinese medical facial mask comprehensive therapy in treating acne vulgaris. Chinese Journal of Integrated Traditional and Western Medicine. 2012;32:624-7.

28. Oberemok SS, Shalita AR. Acne vulgaris, II: treatment. Cutis 2002;70:111-4.

29. Wood L, Egger M, Gluud LL, et al. Empirical evidence of bias in treatment effect estimates in controlled trials with different interventions and outcomes: meta-epidemiological study. BMJ. 2008;336:601-5.

30. Savović J, Jones HE, Altman DG, et al. Influence of re- ported study design characteristics on intervention effect estimates from randomized controlled trials. Ann Intern Med. 2012;157:429-38.

31. Kim CK, Kim DH, Lee MS, et al. Randomized controlled trials on complementary and traditional medicine in the Korean literature. Evid Based Complement Altern Med. 2014;2014:194047.

32. Kleignen J, de Draen AJ, van Everdingen J, Kral L. Placebo effect in double-blind clinical trials: a review of interactions with medications. Lancet. 1994;344:1347-9. Son

33. MJ, Choi SG, Kim YE, Kim YH. Herbal medicines for the treatment of otitis media with effusion: a systematic review of randomised controlled trials. BMJ Open. 2016;6:e011250.

34. Bian Z, Liu B, Moher D, et al. Consolidated standards of reporting trials (CONSORT) for traditional Chinese medicine: current situation and future development. Front Med 2011;5:171-7 